\title{
CANOPY CLASSIFICATION WITH S-BAND POLARIMETRIC SAR DATA
}

\author{
Raffaella Guida ${ }^{1}$, Antonio Natale ${ }^{2,3}$, Rachel Bird ${ }^{4}$, Philip Whittaker ${ }^{4}$, Martin Cohen ${ }^{5}$, David Hall ${ }^{5}$ \\ ${ }^{1}$ Surrey Space Centre, University of Surrey, Guildford, UK \\ ${ }^{2}$ Dipartimento di Ingegneria Biomedica, Elettronica e delle Telecomunicazioni, \\ Università di Napoli Federico II, Napoli, Italy \\ ${ }^{3}$ Istituto per il Rilevamento Elettromagnetico dell'Ambiente (IREA), \\ Consiglio Nazionale per le Ricerche (CNR), Napoli, Italy \\ ${ }^{4}$ SSTL Ltd., Guildford, UK \\ ${ }^{5}$ EADS Astrium, Portsmouth, UK
}

\begin{abstract}
New Synthetic Aperture Radar (SAR) missions in S-band are currently under design but the potential performance of this microwave frequency is still under discussion. This paper presents the outcomes of a study on canopy classification carried out with fully polarimetric S- and Xband datasets contemporaneously acquired by the Astrium airborne SAR demonstrator. Classical polarimetric decompositions have been applied to investigate the S-band capabilities in vegetation monitoring and preliminary results are here presented.
\end{abstract}

Index Terms - S-band, SAR, polarimetry, canopy, classification

\section{INTRODUCTION}

Canopy classification is a well known topic documented by numerous contributions presented in literature. Many significant approaches are based on the use of polarimetric Synthetic Aperture Radar (SAR) images but the issue of the best frequency to adopt for this task is still a matter of discussion to which this paper aims at contributing essentially in three ways. First of all, fully polarimetric SAR images are analyzed in this paper: this means that the information content of all polarimetric channels is relevant to the same acquisition time. Secondly, the images have been contemporaneously acquired both in X- and S-band which allows a better understanding of a frequency (that in S-band which is around $3.2 \mathrm{GHz}$ in our case) very rarely discussed in literature for any application (including canopy classification). Finally, a comparison among datasets acquired at same time but different frequency was made possible. This analysis is of particular importance in a period during which there is a large availability of X-band SAR imagery while S-band SAR missions are under design like the UK spaceborne SSTL NovaSAR-S system [1] or close to be launched as the Chinese HJ-1.

Different fields of applications are likely to be promising in S-band and vegetation is among them. For this purpose, the issues above are addressed here with a focus on canopy classification.

The next section presents the datasets processed for this study and the polarimetric analysis applied.

Section 3 discusses the most interesting results in view of the objectives set and, finally, main conclusions are drawn.

\section{DATA PROCESSING AND POLARIMETRIC ANALYSIS}

The Astrium airborne SAR demonstrator was upgraded to allow the simultaneous acquisition, occurred in summer 2010, of fully polarimetric SAR images in X- and S-band. For a proper use of datasets and comparison of findings, all the images needed to be carefully calibrated considering their polarimetric nature.

We followed the van Zyl procedure described in [2] using the returns from natural targets (assumed to present azimuthal symmetry) and at least one trihedral corner reflector. This technique does not need external calibration targets to correct cross-talk effects (since returns from distributed natural targets are used to infer the cross-talk parameters) but only a trihedral corner reflector for channel imbalance and radiometric calibration.

The datasets were acquired on the site of Marlborough in Southern England. The cross-polarized channel of two acquisitions relevant to geographically adjacent sites is presented in Figure 1. Each original processed channel presented a spatial resolution of $0.35 \mathrm{~m}$ and $0.835 \mathrm{~m}$ respectively in azimuth and slant range. 


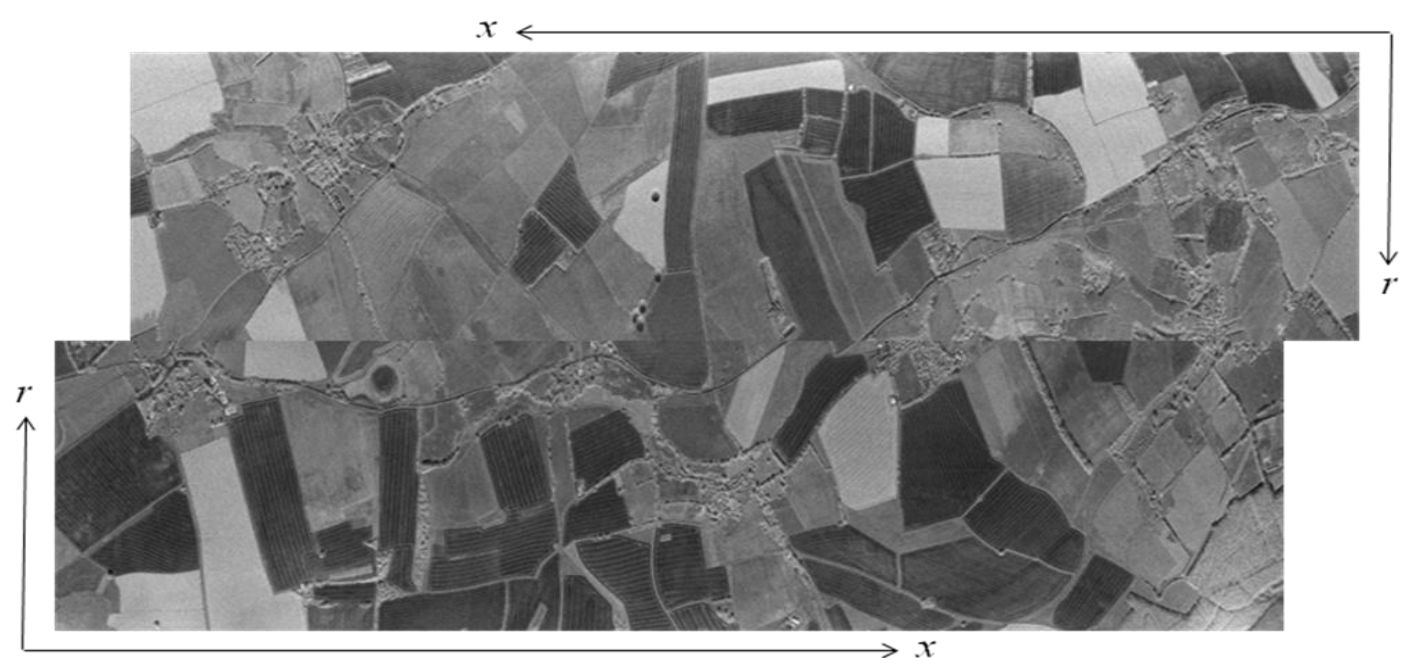

Figure 1 - S-band SAR images of the Marlborough area in HV polarization.

Then a multilook $5 \mathrm{x} 1$ was applied to get a final $1.75 \mathrm{~m} \mathrm{x}$ $1.67 \mathrm{~m}$ resolution in the azimuth/ground range plane when considering an average incidence angle of $30^{\circ}$. After calibration, Pauli and H/alpha decompositions [3] were applied in order to produce the corresponding decompositions maps which are shown for the S-band in Figure 2, the Pauli in (a) and the H/alpha in (b). As regards the Pauli decomposition map, the proper color palette used to represent the different scattering mechanism of volume, surface and double bounce is added in Fig.2(b). Instead, in order to correctly interpret the main outcomes of the H/alpha representation, deriving from the eigenvector-eigenvalue based decomposition, the reader should refer to a different legenda which is also provided in Fig.2(b). Indeed, in Fig.2(b) two parameters are considered, each ranging between different limits. So, each pixel in the H/alpha decomposition map represents the combination of values of the entropy $\mathrm{H}$ and the angle alpha for the same pixel in the original images. These values have been computed considering all the polarimetric channels. Moreover, according to the particular value of the couple $(\mathrm{H}$, alpha), nine different "zones" can be distinguished for land applications according to [4], and we will refer to this distinction in the next section.

Another parameter is usually computed together with $\mathrm{H} / \mathrm{alph}$ and that is the anisotropy. In this study it was computed as well but it seemed to the authors that it did not bring any valuable information content so will not be considered further in this paper. But the interested reader is invited to closer examination of these decompositions in [3]. In Fig.2, the ground truth is also provided for some of the crops in the sites and it is superimposed on the decomposition maps. It is worth to mention that the ground truth was acquired only a couple of weeks after the datasets acquisition, when some farmers were harvesting. This means that discrepancies may be present and unfortunately cannot be removed.

Besides the dataset processed in Figs.1-2, another dataset, still on the Marlborough area and with similar characteristsc, was studied. In total, considering both datasets, six classes were identified and they are wheat (V1), barley (V2), grass (V3), maize (V4), rapeseed (V5) and broad beans (V6). The results in the next section were derived from the analysis carried out on both datasets.

Finally, similar maps have been produced in X-band. For sake of succinctness, they cannot be reported in this paper but will be presented at the conference.

\section{DISCUSSION OF RESULTS}

We remind that we present here a more general discussion of results regarding both Marlborough datasets, which thing explains why, in the table in Fig.3, fields other than those presented in Fig.2 are considered. The results for sixteen fields coming from both datasets are here discussed.

This study lets the authors believe that the S-band frequency can be useful to detect different vegetation crops. As example, it is apparent that barley and maize fields are clearly identified in both maps in Fig.2. Actually, each field associated to (V1, V2, V3, V4) types belongs to one of the well defined representative regions in the H/alpha plane, as summarized in Fig.3 where a table reporting the values of $(\mathrm{H}, \mathrm{alpha})$ and the corresponding "zone" are associated to each vegetation type ("Veg Type") and field number ("Field"). Instead, an amount of uncertainty affects the fields belonging to the types V5 and V6, for which there is not a representative region. But a better classification of canopies 


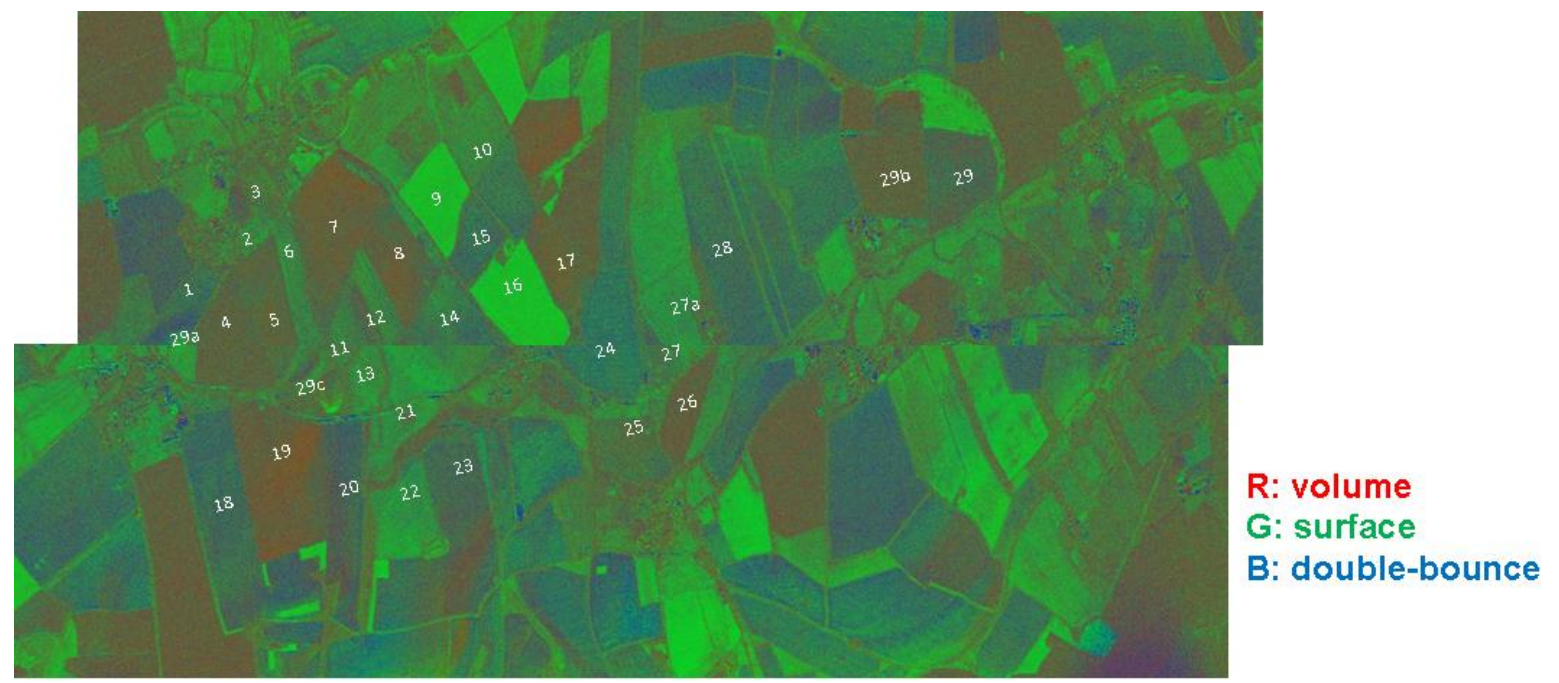

(a)
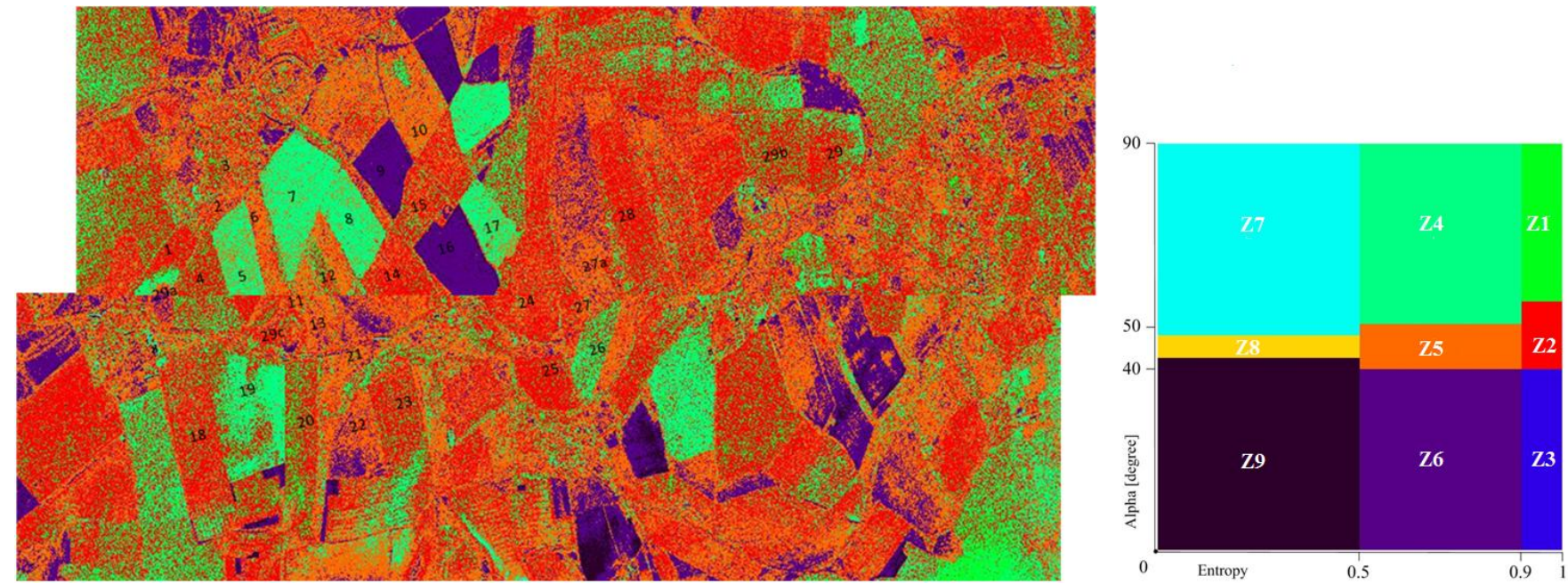

(b)

\section{Ground truth}

\begin{tabular}{|c|c|c|c|c|c|c|c|c|}
\hline Wheat & 8 & Barley & 15 & Wheat & 22 & Grass & 28 & Grass \\
\hline Grass & 9 & Maize & 16 & Maize & 23 & Wheat & 29 & Wheat? \\
\hline Grass & 10 & Grass & 17 & Barley & 24 & Wheat & $29 a$ & Tall grass/flowers \\
\hline Rapeseed & 11 & Grass & 18 & Wheat & 25 & Barley & $29 b$ & Rapeseed \\
\hline Barley & 12 & Grass & 19 & Barley & 26 & Rapeseed & $29 \mathrm{c}$ & Grass \\
\hline Grass & 13 & Grass & 20 & Wheat & 27 & Grass & & \\
\hline Barley & 14 & Grass & 21 & Grass & $27 \mathrm{a}$ & Grass & & \\
\hline
\end{tabular}

Figure 2 - Polarimetric decomposition maps relevant to the dataset in S-band in Fig.1: (a) Pauli, (b) H/alpha. Ground truth is superimposed on both maps

occurs when also the Pauli decomposition is applied. Indeed, for the Pauli decomposition a table similar to the one presented in Fig.3 was produced and we found that in the Pauli space the classes never overlap even if sometimes they are not very distant from each other. A joint use of both decompositions can then improve the overall classification step. Instead, we cannot explain at the moment why the same kind of crop was not always clearly detected. The field 


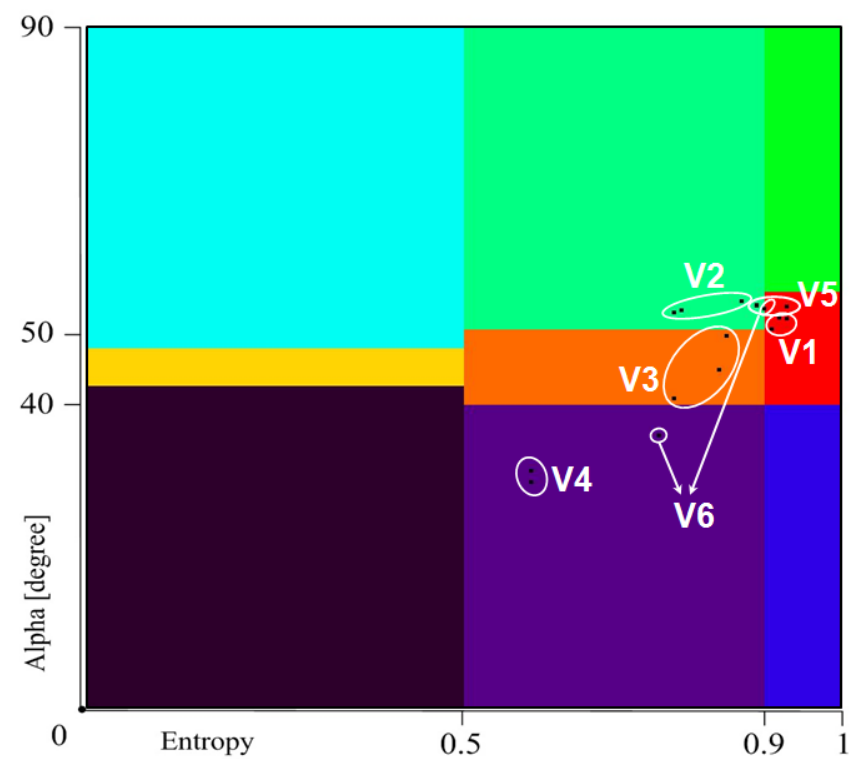

\begin{tabular}{|c|c|c|c|c|c|}
\hline & & & \multicolumn{3}{|c|}{ Cloude } \\
\hline & Field & Veg Type & $\mathrm{H}$ & Alpha & Zone \\
\hline & 18 & V1 & 0.92 & 51.6 & Z2 \\
\hline & 30 & V1 & 0.93 & 51.5 & Z2 \\
\hline & 39 & V1 & 0.91 & 50.1 & $\mathrm{Z2}$ \\
\hline \multirow{13}{*}{$\begin{array}{l}\text { V1: wheat } \\
\text { V2: barley } \\
\text { V3: grass } \\
\text { V4: maize } \\
\text { V5: rapeseed } \\
\text { V6: broadbeans }\end{array}$} & 19 & V2 & 0.87 & 53.8 & $\mathrm{Z4}$ \\
\hline & 7 & V2 & 0.78 & 52.3 & Z4 \\
\hline & 8 & V2 & 0.79 & 52.6 & Z4 \\
\hline & $13-14$ & V3 & 0.84 & 44.7 & Z5 \\
\hline & 32 & V3 & 0.85 & 49.2 & Z5 \\
\hline & 22 & V3 & 0.78 & 40.9 & Z5 \\
\hline & 16 & V4 & 0.59 & 29.8 & Z6 \\
\hline & 9 & V4 & 0.59 & 31.3 & Z6 \\
\hline & $29 b$ & V5 & 0.93 & 53.1 & $\mathrm{Z} 2$ \\
\hline & 26 & V5 & 0.89 & 53.3 & Z4 \\
\hline & $36-37$ & V5 & 0.89 & 53.2 & Z4 \\
\hline & 41 & V6 & 0.90 & 52.8 & $\mathrm{Z} 2$ \\
\hline & 33 & V6 & 0.76 & 36.8 & Z6 \\
\hline
\end{tabular}

Figure 3 - Color code map based on [4] for Figures 2 and mapping of $\mathrm{H} /$ alpha values.

number 25, for instance, which is supposed to be barley, does not seem to present the same scattering as other barley fields like those labeled with number 7 and 8. But, obviously, this can be more easily explained with a not very accurate ground truth.

Analyzing these results in view of the comments given in [4] for each zone, it is interesting to note the clear "violet" signature of the maize. The zone Z6 can be indeed associated to a "surface cover comprising oblate spheroidal scatterers" as stated in [4] which is a very good description of a maize crop.

As regards the X-band data, instead, in all cases a greater confusion was presented in the decomposition maps compared to those in S-band and results will be presented at the conference.

\section{CONCLUSIONS}

This paper presents some of the preliminary results found in a study where S-band fully polarimetric airborne SAR data have been acquired, analyzed and discussed to investigate the potential of future S-band SAR missions. Polarimetric analyses have been applied to two datasets relevant to the same area in the South England with the purpose of investigating S-band capability in distinguishing different kinds of canopies. The first results show that a joint application of Pauli and H/alpha decomposition can produce a good classification of the six different fields analyzed. Apart the specific application, this study certainly shows that this range of frequencies is certainly worth of future studies especially when higher frequencies are likely to fail. We have not yet analyzed the data from a statistic point view which is certainly a due step in the future of this study. Also, the authors are considering the possibility to start a synoptic study in which also lower frequencies are considered and compared. Finally, at the moment further projects have been launched with the purpose of covering other fields of applications for the S-band and they include maritime surveillance and disasters monitoring.

\section{REFERENCES}

[1] P.Whittaker, M.Cohen, D.Hall, L.Gomes "An Affordable Small Satellite SAR System", Proceedings of the $8^{\text {th }}$ IAA Symposium on Small Satellites for Earth Observation, Berlin, 4-8 April 2011.

[2] J.J.van Zyl, "Calibration of polarimetric radar images using only image parameters and trihedral corner reflector responses," IEEE Transactions on Geoscience and Remote Sensing, vol.28, no.3, pp.337-348, May 1990.

[3] J.Lee, E.Pottier, Polarimetric Radar Imaging - From Basics to Applications, CRC Press 2009.

[4] S.R.Cloude, E.Pottier, "An Entropy Based Classification Scheme for Land Applications of Polarimetric SAR", IEEE Transactions on Geoscience and Remote Sensing, vol.35, no.1, pp.68-78, January 1997. 